\title{
Cidofovir for the Treatment of Recurrent Respiratory Papillomatosis: A Review of the Literature
}

\author{
Nadine Shehab, Pharm.D., Burgunda V. Sweet, Pharm.D., and Norman D. Hogikyan, M.D., FACS
}

Recurrent respiratory papillomatosis (RRP) is a rare but potentially severe disease caused by papillomavirus, most often types 6 and 11 . The disease, which occurs in both juvenile and adult forms, is characterized by benign epithelial tumors of the airway that most frequently affect the larynx but can also spread along the entire aerodigestive tract. Recurrent respiratory papillomatosis is the most common benign neoplasm of the larynx in children and the second most frequent cause of childhood hoarseness. Standard treatment, which is palliative only, consists of surgical excision of papillomata to maintain airway patency and improve voice quality. Recurrence despite repeated surgical procedures is the rule. To date, incorporation of adjuvant treatments has not been reliably beneficial in altering the disease course. Several case series have described promising results with cidofovir, a cytosine nucleoside analog with antiviral activity. To evaluate the data available on the safety and efficacy of cidofovir for the treatment of RRP, we conducted a MEDLINE search for all case reports or series from January 1966-August 2004 describing cidofovir therapy in either adults or children with RRP. The bibliographies of qualifying articles were also searched for relevant references. In both adults and children with mild-to-severe RRP, intralesional administration of cidofovir directly into the site of papillomata was associated with partial-to-complete regression of papillomata, improvement in voice quality and airway status, and decreased need for surgery. Wide variation in intralesional cidofovir dose (2-57 mg), frequency (every 2-8 wks), and duration ( 4 mo-4 yrs) was found. Successful outcomes have also been reported with intravenous cidofovir, but data are limited to three case reports. Rash, headache, and precordialgia were the only adverse effects reported with intralesional cidofovir. Nephrotoxicity and neutropenia secondary to either intralesional or intravenous cidofovir were not observed. Long-term risks associated with intralesional administration remain to be seen. Further studies are necessary to determine the most appropriate dose, frequency, and duration of therapy, and to fully characterize the safety profile of cidofovir when given intralesionally.

Key Words: recurrent respiratory papillomatosis, cidofovir. (Pharmacotherapy 2005;25(7):977-989)

\section{OUTLINE}

Epidemiology

Transmission

Clinical Features

Management

Efficacy

Children
Adults

Children and Adults

Percutaneous Intralesional Cidofovir Treatment Intravenous Cidofovir Treatment

Safety

Dosage and Duration of Therapy

Limitations of the Literature

Conclusion 
Recurrent respiratory papillomatosis (RRP) is a rare disease caused by human papillomavirus (HPV), most commonly types 6 and $11.1^{1,2}$ Papillomavirus is a small, nonenveloped virus consisting of an icosahedral capsid enclosing a double-stranded, circular DNA genome. At least $70 \mathrm{HPV}$ types have been identified, most of which are associated with epithelial tumors of the skin and mucous membranes, such as plantar warts, condylomata acuminata (anogenital warts), and epidermodysplasia verruciformis. ${ }^{3,4}$ Recurrent respiratory papillomatosis is manifested as exophytic lesions that most frequently affect the larynx but can also spread along the entire aerodigestive tract. The presence of these benign neoplasms can cause symptoms ranging from dysphonia to life-threatening respiratory distress, and can profoundly affect the quality of life of patients with RRP., 6

Traditionally, RRP has been classified based on patient age at diagnosis, such that two forms of the disease have been described: one with onset in childhood, which is arbitrarily defined as younger than 12 years (juvenile-onset RRP), the other in adulthood (adult-onset RRP). Juvenileonset RRP is observed more often and is typically more aggressive than its adult counterpart. Peak age for the juvenile form is around 4 years, compared with the third decade of life for the adult form. ${ }^{7,8}$ Among children, RRP is the most common benign neoplasm of the larynx and the second most frequent cause of hoarseness. ${ }^{9}$

\section{Epidemiology}

Accurate epidemiologic data for RRP are not available. The incidence of RRP among children in the United States was estimated at 4.3/100,000 children, based on a survey questionnaire administered in 1993 to board-certified U.S. otolaryngologists. ${ }^{10}$ The incidence among patients aged 15 years or older was estimated at 1.8/100,000. Each year, 1500-2500 new cases of juvenileonset RRP and 3600 new cases of adult-onset

From the Departments of Pharmacy Services (Drs. Shehab and Sweet) and Otolaryngology (Dr. Hogikyan), and the Vocal Health Center (Dr. Hogikyan), University of Michigan Health System, University Hospital, Ann Arbor, Michigan; and the College of Pharmacy (Drs. Shehab and Sweet), the Medical School (Dr. Hogikyan), and the School of Music (Dr. Hogikyan), University of Michigan, Ann Arbor, Michigan.

Address reprint requests to Nadine Shehab, Pharm.D., Department of Pharmacy Services, University of Michigan Health System, University Hospital, 1500 East Medical Center Drive, Room B2D301, Ann Arbor, MI 48109-0008; e-mail: nshehab@umich.edu.
RRP are diagnosed in the United States. The annual cost of managing juvenile-onset RRP is \$40-123 million. ${ }^{6}$ Males and females appear to be nearly equally affected by juvenile-onset RRP in contrast with adult-onset RRP, which preferentially affects men. ${ }^{11}$

\section{Transmission}

The mode of HPV transmission resulting in RRP is not well understood. Studies have indicated that similar HPV types are associated with respiratory papillomatosis and condylomata acuminata, and that at least $50 \%$ of mothers of children with RRP have a history of genital tract HPV disease. ${ }^{12-16}$ This led to the hypothesis that juvenile-onset RRP is acquired through vertical transmission. In particular, it has been suggested that birth to young primiparous mothers with condylomata has been a risk factor for juvenileonset RRP. ${ }^{15,16}$ However, in utero transmission of the disease cannot be excluded and has been suggested in reports of neonatal papillomatosis. ${ }^{17-19}$ Adult-onset RRP has been anecdotally associated with a higher than expected number of lifetime sexual partners and with oral-genital contact. ${ }^{20}$ It is indeed evident that a clearer understanding of the mode of RRP transmission is needed.

\section{Clinical Features}

The hallmark clinical manifestation of RRP in children has been described as a triad of relentlessly progressive hoarseness, stridor, and respiratory distress. ${ }^{11}$ Chronic cough, recurrent pneumonia, and failure to thrive may also occur. Given these symptoms, a misdiagnosis of asthma often leads to a delay in determining the true airway pathology. Progressive hoarseness is almost universally the primary symptom in adults. Airway symptoms may occur in those with advanced or aggressive disease but are much less common than in pediatric patients. Extralaryngeal spread of respiratory papillomata (most frequently to the oral cavity, followed by the trachea and bronchi) has been identified in approximately $30 \%$ of children and $16 \%$ of adults with RRP. In the presence of aggressive disease, spread of the papillomata into the tracheobronchial tree and/or pulmonary parenchyma can be fatal. ${ }^{10,21,22}$

Recurrent respiratory papillomatosis remains a very frustrating disorder for patients and for surgeons eager to perform a curative intervention. Recurrence despite repeated surgery is the rule, and to date, incorporation of adjuvant treatments 
has not been reliably beneficial in altering the disease course. ${ }^{10}$ Recurrence has been attributed to the persistence of latent virus in nondiseased sites adjacent to areas that are clinically affected by HPV. ${ }^{23}, 24$ Frequency of recurrence is highly unpredictable, with extreme interpatient variability in the disease course. Intrapatient variability also occurs, but is less striking than interpatient variability.

Malignant conversion of previously benign papillomatous lesions is rare, occurring in $3-5 \%$ of patients with RRP. ${ }^{25}$ Malignant conversion is most often associated with HPV types 16 and 18, which have high malignant potential. Exposure to radiation and cytotoxic therapies (e.g., methotrexate) are risk factors for malignant transformation. $^{26-31}$ However, degeneration of previously benign papillomata to squamous cell carcinoma also can occur independent of any such risk factors. ${ }^{32-34}$

\section{Management}

The current standard of care for RRP management consists of surgical excision of respiratory tract papillomata. For patients with potential for significant airway compromise, the primary goal of treatment is improvement and maintenance of an adequate airway. For patients without airway symptoms, the focus of treatment is improvement of voice quality. Given that cure is rare and repeated procedures are expected, surgical treatment decisions usually factor in the existing pathology, potential for clinical decline, and desire to extend the interval between operations, if possible.

Papillomata typically are excised by direct microlaryngoscopy and with the patient under general anesthesia. Specific excision techniques vary and include traditional cold microlaryngeal instruments, laser (most often carbon dioxide) and, more recently, the microdebrider. ${ }^{35,36}$ For patients with severe symptomatic disease, frequent surgical intervention (as often as every 2-4 wks) may be necessary. Repeated surgery in the same anatomic site increases the risk of complications. As many as $21-45 \%$ of patients experience complications, such as mucosal scarring, webbing, impaired vocal cord motion, and subglottic or glottic stenosis. ${ }^{37-40}$ These complications can affect voice quality and airway patency. In addition, surgical trauma may induce new papilloma growth. ${ }^{41}$ Patients with aggressive disease may require a tracheotomy to maintain an airway; this, in turn, has been associated with subsequent development of tracheal and stomal papillomata. ${ }^{37,42}$ If possible, tracheotomy should be avoided in patients with RRP.

Surgery alone rarely cures RRP and is probably best thought of as symptomatic treatment since it does not address the underlying viral infection. Approximately $10 \%$ of patients with more aggressive disease require some form of adjuvant therapy. ${ }^{11}$ Suggested criteria for starting adjuvant therapy are the need for more than four surgical procedures/year, distal multisite spread of the disease, and rapid regrowth of papilloma disease with airway compromise. ${ }^{11}$ Therapeutic modalities used as adjunctive treatment for RRP are antivirals (cidofovir, acyclovir, ribavirin), bleomycin, cimetidine, interferon $\alpha-2 \mathrm{a}$ and $-2 \mathrm{~b}$, indole-3carbinol-diindolylmethane, methotrexate, photodynamic therapy, and retinoids (cis-retinoic acid, isotretinoin). ${ }^{43-67}$ However, none of these approaches has been extensively studied, and no single therapy or agent has consistently been effective in eradicating RRP. Interferon is one of the more commonly studied adjuvant therapies, but its use is limited by its unfavorable side effect profile and high rate of disease resurgence on discontinuation of treatment. ${ }^{51-57}$

Owing to the viral etiology of RRP, acyclovir, a guanosine nucleoside analog, and cidofovir, a cytosine nucleoside analog, have been studied as adjuvant treatments for RRP. One study described the ideal antiviral drug for papillo-mavirus infections as one that would eliminate existing lesions, eradicate latent populations of viral DNA, and allow for the development of natural immunity to the virus. ${ }^{4}$ The rationale for the use of acyclovir is less clear. Unlike cidofovir, the antiviral activity of acyclovir depends on the presence of virus-specific thymidine kinase for phosphorylation to its active metabolite. ${ }^{68}$ This enzyme is not encoded by papillomavirus. ${ }^{4}$ Results of small studies examining the efficacy of acyclovir for patients with RRP have been conflicting. ${ }^{43-45}$ Acyclovir may be effective for RRP in the presence of coinfection with a virus capable of activating acyclovir, such as herpes simplex virus (HSV) or cytomegalovirus (CMV). ${ }^{43}$

Reports of intralesional cidofovir show more promising results. Cidofovir [(S)-1-3-hydroxy-2(phosphonylmethoxy)propyl]cytosine (HPMPC)] is a prodrug that undergoes intracellular phosphorylation to its active metabolite, cidofovir diphosphate (HPMPCpp), which then competitively inhibits incorporation of deoxycytosine-5'triphosphate by viral DNA polymerase into the viral DNA strand in a way that impedes viral 
Table 1. Characteristics of Intralesional Cidofovir Treatment in Children and Adults with Recurrent Respiratory Papillomatosis

\begin{tabular}{|c|c|c|c|c|c|c|}
\hline \multirow[b]{2}{*}{$\begin{array}{l}\text { No. of } \\
\text { Patients }\end{array}$} & \multicolumn{5}{|c|}{ Mean (range) } & \multirow[b]{2}{*}{$\begin{array}{l}\text { Frequency of } \\
\text { Injections }\end{array}$} \\
\hline & $\begin{array}{l}\begin{array}{l}\text { Age } \\
(\text { yrs })^{\mathrm{a}}\end{array} \\
\end{array}$ & $\begin{array}{l}\text { Severity } \\
\text { Score }^{\mathrm{a}, \mathrm{b}}\end{array}$ & $\begin{array}{l}\text { Total Dose } \\
(\mathrm{mg})^{\mathrm{c}}\end{array}$ & $\begin{array}{c}\text { No. of } \\
\text { Injections }\end{array}$ & $\begin{array}{c}\text { Duration of } \\
\text { Therapy (mo) }\end{array}$ & \\
\hline $10^{77-79}$ & $4(2-9)$ & $18^{g}$ & NA (2.5-20.0) & $8(4-13)^{g}$ & $7.5(3.8-13.5)$ & Every 2-3 wks \\
\hline $1^{79}$ & 9 & 20 & NA (10.0-20.0) & 28 & 51.0 & NA \\
\hline $4^{82}$ & $4(3-7)$ & NA & $8.3(2.0-15.0)$ & $6(6-7)$ & $7.5(7.0-8.0)$ & Every $6-8$ wks \\
\hline $\begin{array}{l}6^{83, \mathrm{~h}} \\
5^{83, \mathrm{i}}\end{array}$ & $\begin{array}{l}7(2-14) \\
7(4-10)\end{array}$ & $\begin{array}{l}12(8-20) \\
16(8-25)\end{array}$ & $\begin{array}{l}5.6(1.0-15.0) \\
18.5(10.0-30.0)\end{array}$ & $\begin{array}{l}4 \\
8\end{array}$ & $\begin{array}{l}4.0 \\
4.0\end{array}$ & $\begin{array}{l}\text { Monthly } \\
\text { Monthly }\end{array}$ \\
\hline $17^{84}$ & $44(11-77)$ & NA & $17.9(2.5-42.5)$ & $7(2-15)$ & $4.7(1.0-13.0)$ & Every 2 wks \\
\hline $13^{85}$ & $48(18-85)$ & $10(2-29)$ & $22.5(2.5-37.5)$ & $6(1-19)$ & $\begin{array}{l}13.0 \text { ( } 7.3 \text { days- } \\
3.7 \text { yrs) }\end{array}$ & Monthly \\
\hline 10 JORRP $^{86}$ & $13(5-25)$ & 10 & NA & $6(2-20)$ & $13.8(\mathrm{NA})$ & Monthly \\
\hline 16 AORRP $^{86}$ & $37(25-56)$ & 5 & & $4(1-7)$ & 9.9 (NA) & Monthly \\
\hline $5^{88}$ & 44 & NA & $47.0(38.0-57.0)$ & $7(2-12)$ & $12.0(7.0-16.0)$ & Every 2-4 wks \\
\hline
\end{tabular}

$\mathrm{NA}=$ not available; JORRP = juvenile-onset recurrent respiratory papillomatosis; AORRP = adult-onset recurrent respiratory papillomatosis.

${ }^{\mathrm{a}}$ At onset of cidofovir injections.

${ }^{b}$ Reflects anatomic assessment of the papilloma stage as determined by the scoring system in reference. ${ }^{89}$

'Total cidofovir dose/patient/session.

${ }^{\mathrm{d}}$ Refers to total number of injections/patient over entire treatment period.

${ }^{e}$ Complete responders had no visible lesions, had a severity score of 0 , or were disease free at end of cidofovir protocol or at follow-up.

${ }^{\mathrm{f}} \mathrm{Partial}$ responders had residual, relapsed, or recurrent disease at end of cidofovir protocol or at follow-up

gData were available for 9 of 10 patients.

hPatients received a single series of cidofovir 5-mg/ml injections.

${ }^{i}$ Patients with recurrent or recalcitrant RRP received an additional series of cidofovir $10-\mathrm{mg} / \mathrm{ml}$ injections

DNA synthesis. ${ }^{68,69}$ The precise mechanisms by which cidofovir exerts its inhibitory effects on HPV are not understood. Cidofovir also possesses activity against other viruses (e.g., HSV and CMV). However, unlike these, HPV relies on the host cell DNA polymerase, and not a virally encoded polymerase, for replication. ${ }^{4,} 70$ Induction of apoptosis and augmentation of hostmediated immune responses have been proposed as potential mechanisms of action of cidofovir in RRP. $^{70-72}$ Also, cidofovir may impede the carcinogenesis of HPV-infected cells by downregulating the expression of oncoproteins, leading to restoration of tumor suppressor pathways. ${ }^{73,74}$

Cidofovir demonstrates broad antiviral activity against herpes viruses, such as thymidinekinase-producing and deficient HSV-1 and HSV2, varicella-zoster virus, CMV, Epstein-Barr virus, human herpes viruses (types 6, 7, and 8), polyomavirus, papillomavirus, adenovirus, and poxviruses (e.g., variola virus, cowpox virus, monkeypox virus, camelpox virus, vaccinia virus, Orf virus, and molluscum contagiosum). ${ }^{75}$ Cidofovir is approved by the Food and Drug Administration for treatment of CMV retinitis in patients with acquired immunodeficiency syndrome. ${ }^{76}$

To evaluate the data available on the safety and efficacy of cidofovir for the treatment of RRP, we conducted a MEDLINE search for all case reports or series from January 1966-August 2004 describing cidofovir therapy in either adults or children with RRP. The bibliographies of qualifying articles were also searched for relevant references.

\section{Efficacy}

The first reported use of intralesional cidofovir injections to treat RRP was described in a 69year-old woman with squamous papilloma in the hypopharynx and esophagus. ${ }^{72}$ Much of the data since that report have been limited to case series in which cidofovir was similarly administered as an intralesional injection directly into the site of each papilloma in adults and children with mildto-severe RRP (Table 1). ${ }^{77-87}$ In all these reports, intralesional cidofovir injections were administered to patients who received general anesthesia. In addition, one case series reported office-based treatment with percutaneous intralesional cidofovir injections. ${ }^{88}$ With the exception of a few reports, cidofovir was used as an adjuvant therapy to surgery or in combination with other adjuvant agents.

\section{Children}

Investigators evaluated the efficacy of intralesional cidofovir in five pediatric patients who 
Table 1. (continued)

\begin{tabular}{lcc}
\hline \multicolumn{3}{c}{ No. (\%) of Patients } \\
\hline $\begin{array}{c}\text { Complete } \\
\text { Responders }^{\mathrm{e}}\end{array}$ & $\begin{array}{c}\text { Partial } \\
\text { Responders }^{\mathrm{f}}\end{array}$ & Nonresponders \\
\hline $5(50)$ & $5(50)$ & 0 \\
0 & $1(100)$ & 0 \\
$1(25)$ & $2(50)$ & $1(25)$ \\
$6(100)$ & 0 & 0 \\
$3(60)$ & 0 & $2(40)$ \\
$9(53)$ & $6(35)$ & $2(12)$ \\
$13(100)$ & - & - \\
$2(20)$ & $7(70 \%)$ & $1(10 \%)$ \\
$6(37)$ & $10(63 \%)$ & 0 \\
0 & $5(100 \%)$ & 0 \\
\hline
\end{tabular}

received cidofovir $2.5-5.0 \mathrm{mg} / \mathrm{ml}$ every $2-3$ weeks for 4-13 months. ${ }^{77}$ Patients received 4-13 cidofovir injections every 2-3 weeks in conjunction with surgical debulking and carbon dioxide laser treatment. Therapy was continued until regression in papilloma growth was observed. In follow-up reports, the long-term outcome for these initial five patients was reported along with results for five new patients who received four intralesional injections of cidofovir $5 \mathrm{mg} / \mathrm{ml}$ every 2 weeks, and one additional patient who received 28 injections over a 51-month period. ${ }^{78,79}$ All of these children (aged 2-9 yrs) had aggressive RRP unresponsive to adjuvant medical therapy and required operative intervention every 2-6 weeks. Severity of their papillomatosis was graded using a scoring system that entailed numeric grading $(0$ = absent, 1 = surface lesion, 2 = raised lesion, 3 = bulky lesion). ${ }^{89}$ The extent of papilloma growth was graded at 25 predefined aerodigestive subsites such that 75 (i.e., a score of 3 at all 25 subsites) was the maximum allowable score. During each session, the total dose of cidofovir administered was $2.5-20 \mathrm{mg}$.

Complete remission, defined as the absence of gross disease diagnosed by flexible endoscopy or operative direct laryngoscopy, was observed in 5 of 11 patients who received a mean of 9.8 (range 4-13) injections over 5-9 months. Of the remaining children, five continued to have active disease but no longer required cidofovir injections to maintain patent airways. These five children received a mean of five (range 4-8) injections. Their mean RRP severity score decreased from 17.8 (range 11-26) to 4.0 (range
2-6). Of the entire cohort, one patient-who was described as having recalcitrant disease and an initial severity score of 20-showed a less favorable response to cidofovir. This was demonstrated by the recurrence of papillomata on discontinuation of intralesional injections. After receiving 28 injections over a 51-month period, the child's severity score was 7 . The authors of this case series reported the longest mean follow-up time in the literature $(37$ mo [range $7-66 \mathrm{mo}$ ]). ${ }^{79}$ Five patients in this case series remained disease free for a mean of 51.6 months (range 39-66 mo).

The efficacy of cidofovir was also evaluated in a small cohort of four pediatric patients (average age $51 \mathrm{mo}$ ) with severe RRP requiring at least six surgical excisions/year. ${ }^{82}$ Patients who had received treatment with adjuvant chemotherapy within the preceding 6 weeks were excluded from the study. At the time of endoscopic debridement, cidofovir was administered at a concentration of $5 \mathrm{mg} / \mathrm{ml}$ into sites in which all visible papillomata were being excised. A series of six operations with cidofovir injections were performed on each patient. Each surgery was performed 6-8 weeks apart over a period of 7 (three patients) or 8 (one patient) months. Efficacy was evaluated throughout the treatment period and was based on the change in severity scores as defined by the grading system used. ${ }^{89}$

At 15-month follow-up, only one of the four patients had complete resolution of disease, described as the absence of papillomata and clinical symptoms of RRP. ${ }^{82}$ Two patients experienced an initial decrease in the severity and/or extent of disease. However, recurrence of disease to pretreatment stages required further frequent surgical intervention. One patient with diffuse disease affecting the trachea experienced no resolution in growth of either the laryngeal or the tracheal papillomata. The author concluded that although intralesional cidofovir was beneficial in temporarily reducing the progression of RRP, the benefit was no longer evident once treatment was discontinued.

A prospective, nonrandomized case series examined the effect of intralesional injections of cidofovir, as an adjuvant to carbon dioxide laser therapy, on the disease severity score of pediatric patients with mild-to-severe RRP. ${ }^{83}$ Eleven patients aged 2-14 years completed a series of four monthly injections of cidofovir $5 \mathrm{mg} / \mathrm{ml}$ (injection volume $0.2-3.0 \mathrm{ml}$ ). Patients who had recurrent or recalcitrant RRP 1 month after the end of the initial series of injections were 
subsequently treated with an additional series of four monthly injections at an increased concentration of $10 \mathrm{mg} / \mathrm{ml}$ (injection volume $1.0-3.0 \mathrm{ml}$ ). Disease severity and extent of subsite involvement were assessed using the scoring system described above.

At the first follow-up period, 1 month after the last injection, significant improvements in the absolute and the percent change in severity and subsite scores were observed across the 11 patients. Seven (64\%) had a complete (severity score 0 ) or nearly complete (severity score 1) response. This response was maintained in six $(86 \%)$ of these seven patients at a mean \pm SD follow-up of $9.3 \pm 7.2$ months after the last cidofovir injection. Five patients required treatment with the second series of four injections. Three of these five experienced complete resolution of RRP; disease severity scores remained unchanged in two patients with moderate or advanced disease. Mean time to follow-up was 2 months (range 1-4 mo) after the last cidofovir injection in the second series.

Although the results of this case series suggested diminished treatment response in patients with more severe disease, a positive correlation between baseline disease severity scores and treatment response was not evident by statistical analysis (Spearman correlation coefficient $-0.62,95 \%$ confidence interval -1.0 to -0.03). The authors concluded that intralesional injections of cidofovir were effective at reducing disease burden in children with RRP, but that higher concentrations may be necessary for more advanced disease, and patients with more severe disease at baseline may be more resistant to therapy.

\section{Adults}

In two case series describing intralesional cidofovir in adults, ${ }^{84,85}$ therapy was associated with partial to complete remission of RRP in most patients. In the first report, 17 patients aged 11-77 years (mean 44 yrs) received 2-15 (mean 7.2) intralesional injections of cidofovir $2.5 \mathrm{mg} / \mathrm{ml}$ (injection volume $1-12 \mathrm{ml}$ ). ${ }^{84}$ All patients had biopsy-proven severe laryngeal RRP. Initially, cidofovir was administered every other week until a clinical response was noted, and at a reduced frequency thereafter. Two additional sets of injections were administered at the previous site of lesions when the last macroscopic lesion had disappeared. Follow-up consisted of indirect laryngoscopy performed every 4 weeks on an outpatient basis.

Intralesional cidofovir therapy was successful in 14 patients. Complete remission, with no evidence of relapse, was observed in 9 of the 14 patients after a mean treatment duration of 3.3 months (range 1-8 mo). Mean duration of remission after the end of treatment in these nine patients was 13.6 months $(2-27 \mathrm{mo})$. The remaining five patients who responded successfully to therapy required additional injections for relapsed lesions throughout their treatment course. Complete disease regression was observed in two of these five, at 6 and 7 months, respectively, after the last cidofovir injection. Of the remaining three, one progressed during cidofovir therapy after an initial marked response, one had partial remission and remained stable for more than 1 year after the last injection, and one was lost to follow-up after four injections.

The authors concluded that intralesional cidofovir demonstrated a clear effect on the progression of papillomatosis tumors, and that their findings warrant further examination of the effect of cidofovir on long-term outcomes in patients with RRP.

In the second case series, the efficacy of intralesional cidofovir in adults with RRP was evaluated in an open-label, prospective study. ${ }^{85}$ Thirteen patients with biopsy-proven laryngeal papillomatosis limited to the glottis, supraglottis, or subglottis received monthly injections of intralesional cidofovir until intraoperative evaluation revealed no evidence of visible papilloma. Patients were then monitored for recurrent disease with video laryngeal examination every 3 months. Excisional surgery was not performed during cidofovir therapy. Cidofovir was administered at a concentration of 4.17-6.25 $\mathrm{mg} / \mathrm{ml}$ (injection volume $0.6-6 \mathrm{ml}$, mean $3.8 \mathrm{ml}$ ). At study completion, all patients had achieved clinical remission, with no visible papilloma. Patients were treated for a mean of 1.11 years (range 7.3 days-3.7 yrs); lesion remission was achieved after a mean of six injections (range 1-19).

Statistical analysis demonstrated a significant, positive correlation between the number of injections required until lesion remission was achieved and both duration $(\mathrm{p}=0.005)$ and severity of disease $(p=0.05)$ before cidofovir treatment was started. The authors concluded that intralesional injection of cidofovir is an excellent treatment option, but noted that its long-term efficacy in achieving remission requires further study. 


\section{Children and Adults}

In the largest series of patients to undergo adjuvant treatment with intralesional cidofovir, ${ }^{86}$ 26 patients, both adults and children with mildto-severe biopsy-proven RRP, received at least three monthly intralesional injections of cidofovir $5 \mathrm{mg} / \mathrm{ml}$. In addition, surgical debulking with cold steel instruments or carbon dioxide laser vaporization was performed in the setting of airway obstruction or resistance to cidofovir therapy. Resistance was identified by persistence of a lesion despite cidofovir treatment. The cidofovir protocol was started in patients with adult-onset RRP (mean age $37 \mathrm{yrs}$ ), and in those with juvenile-onset RRP (mean age 13 yrs). The volume injected during each procedure varied by patient and was not reported. Disease severity was based on the same grading system as in the previous case series discussed. Patients with juvenile-onset RRP had more severe and diffuse disease than their adult counterparts. This was demonstrated by their mean severity scores at baseline (juveniles 10.4, adults 5.1) and the number of operations they had undergone before cidofovir therapy.

At study completion, eight (31\%) of the 26 patients were disease free, 17 (65\%) showed significant improvement, and 1 (4\%) patient with juvenile-onset RRP showed no improvement in initial severity score despite six cidofovir injections. The reduction from baseline in mean severity score was $75 \%$ (from 5.1 to 1.3 ) in the 16 patients with adult-onset RRP and 76\% (from 10.4 to 2.5 ) in the 10 with juvenile-onset RRP. However, the patients with juvenile-onset RRP required a mean of six cidofovir injections (range 2-20) to achieve a similar extent of improvement as those with adult-onset RRP, who received a mean of four injections (1-7).

The authors discussed factors that may have positively influenced the response to cidofovir in their patient population: older age at onset of RRP (i.e., a diagnosis of adult-onset RRP), papilloma growth at supraglottic subsites in adults and glottic subsites in children, patient adherence to the protocol, total number of injections, and concomitant surgical excision. Neither local nor systemic side effects secondary to cidofovir therapy were noted throughout the study or during the follow-up period, which was a mean of 8 months (range $1-35 \mathrm{mo}$ ).

Percutaneous Intralesional Cidofovir Treatment

In the only case series to describe office-based cidofovir treatment, five men with severe laryngeal papillomatosis received percutaneous intralesional cidofovir injections. ${ }^{88}$ The treatment goal was to minimize the need for laser surgery under general anesthesia. Before the start of cidofovir treatment, patients had required operative intervention every 2.8 months on average. Cidofovir injections were initially administered every 2-4 weeks, then at longer intervals depending on therapeutic response. All five patients underwent laser ablation of papillomata at the beginning of percutaneous treatments.

Although papilloma volume was reduced in all patients, none experienced complete remission, and two of the five subsequently required operative intervention. Residual papillomatous lesions were present in the anterior commissure in one of these two patients and in the left aryepiglottic fold of the other; both required further surgical treatment. The authors suggested that percutaneous intralesional cidofovir injections may be useful as adjunct therapy, but not as a replacement for laser ablation in adult patients with papillomata involving the vocal cords and anterior commissure.

\section{Intravenous Cidofovir Treatment}

Three case reports described systemic rather than intralesional administration of cidofovir for treatment of RRP. ${ }^{90-92}$ In the first report, a 35year-old woman with a history of laryngeal papillomatosis diagnosed at infancy was having daily episodes of hemoptysis and progressive dyspnea on exertion. ${ }^{90}$ She had already undergone treatment with a total of 96 resections and laser ablations. Computed tomography of the thorax revealed several bilateral pulmonary nodules and cysts. A presumptive diagnosis of HPV-related multicystic lung disease was made, and treatment with intravenous cidofovir $5 \mathrm{mg} / \mathrm{kg}$ was begun. Cidofovir was administered biweekly in conjunction with probenecid for 2 months, followed by aggressive hydration to minimize the risk of cidofovir-associated nephrotoxicity. In anticipation of cessation of cidofovir therapy, interferon $\alpha-2 \mathrm{a}$ was started.

After 9 months of concomitant cidofovir and interferon therapy, the patient developed alopecia and leukopenia necessitating discontinuation of interferon and a dose reduction in cidofovir. Cidofovir $4 \mathrm{mg} / \mathrm{kg}$ was continued every 2 weeks for an additional 12 weeks and was stopped after the patient had received a total of 51 doses over 24 months. Eighteen months after cessation of 
all therapy, the patient was doing well, as evidenced by normal pulmonary function test results, stabilization of parenchymal disease, and the need for only one laser surgical treatment.

The second report described the administration of intravenous cidofovir in a 34-year-old woman with diffuse RRP extending from the larynx to the trachea. ${ }^{91}$ Her disease was refractory to repeated excisional surgery and a 4-year course of interferon $\alpha-2 b$ therapy. The patient had undergone more than 200 microlaryngoscopies since the age of 7 years and suffered from voice problems and recurrent pneumonia due to bronchial obstruction. Interferon $\alpha-2 b$ therapy was discontinued, and therapy was started with intravenous cidofovir $5 \mathrm{mg} / \mathrm{kg}$ once/week for 2 weeks, followed by $5 \mathrm{mg} / \mathrm{kg}$ every 2 weeks. Treatment with cidofovir was continued for 2 months before interferon $\alpha-2 b$ was reinstituted due to recurrence of papillomatosis in the larynx. One year after combination therapy, examination indicated complete macroscopic disappearance of the patient's laryngeal lesions, marked response of the papillomata in the trachea, and regression of the endobronchial lesions and intrapulmonary consolidations.

The third case report described an 8.5-year-old girl with RRP of the pharynx, larynx, trachea, and lungs. ${ }^{92}$ Intravenous cidofovir $5 \mathrm{mg} / \mathrm{kg}$ was administered over 1 hour with the following schedule: once/week for 4 weeks, every other week for 8 weeks, every third week for 9 weeks, every 4-6 weeks for 21 weeks and, in the last month, once/week for 4 weeks. In addition, oral probenecid $25 \mathrm{mg} / \mathrm{kg}$ and intravenous hydration were administered before and after each cidofovir dose. Cidofovir was given as a single therapy for 4 months before surgical treatment with carbon dioxide laser became necessary to preserve airway patency. Also, adjuvant therapy with indole-3-carbinol was begun after 14 doses of cidofovir.

Disappearance of the pharyngeal papillomata was noted at 6 months, bronchial papillomata at 8 months, and laryngeal papillomata at 9 months after the start of cidofovir therapy. Progression of tracheal lesions was reduced after 10 months of therapy. No local or systemic adverse effects secondary to cidofovir were observed throughout the 12-month treatment period.

\section{Safety}

The most common and severe adverse effects of systemically administered cidofovir are nephrotoxicity and neutropenia. ${ }^{76}$ With the exception of one patient who developed leukopenia while receiving interferon and intravenous cidofovir concomitantly, these adverse effects have not been reported in the literature describing the use of cidofovir for RRP. ${ }^{90}$ Given the low doses administered in the treatment of RRP and the local nature of administration, the likelihood of these systemic toxicities resulting from intralesional cidofovir therapy is minimal.

To assess the extent of systemic absorption of intralesional cidofovir, a group of investigators measured serum cidofovir concentrations in three patients receiving intralesional injections of cidofovir every other week. ${ }^{84}$ A total of five series of cidofovir serum concentrations were obtained at various times, and up to 48 hours after the end of the cidofovir injection. Serum cidofovir was detectable in two of the three patients 5-15 minutes after the intralesional injection; the highest and lowest concentrations were 0.6 and $0.36 \mu \mathrm{g} / \mathrm{ml}$, respectively. These concentrations are considerably lower than those achieved with systemic administration of cidofovir. Pharmacokinetic studies have reported peak serum cidofovir concentrations of 7 and 24 $\mu \mathrm{g} / \mathrm{ml}$ after 1 -hour cidofovir infusions of 3 and $10 \mathrm{mg} / \mathrm{kg}$, respectively. ${ }^{93}$

The safety of intralesional cidofovir injections has also been evaluated in animals. ${ }^{94}$ Canine subepithelial vocal folds were injected biweekly for 6 months with cidofovir 2.5-37.5 mg (injection volume $0.5 \mathrm{ml}$ ). At the end of the 6month injection period, dose-dependent histopathologic changes noted were endomysial edema with muscle fiber separation, atrophy, and scarring of vocal folds. Onset of atrophy and scarring was observed after 3,7 , and 11 injections in the vocal folds with cidofovir 3.5, 20 , and $10 \mathrm{mg}$, respectively.

Six months after the end of the last injection, resolution of histologic abnormalities was complete in the vocal folds of animals given lowdose cidofovir $2.5 \mathrm{mg}$, nearly complete in those given intermediate-dose cidofovir 5 or $10 \mathrm{mg}$, and not apparent in those given high-dose cidofovir 20 or $37.5 \mathrm{mg}$. Complete blood counts and renal parameters were unchanged throughout the injection period. The investigators concluded that intralaryngeal cidofovir in animals leads to dose-dependent scarification of the vocal folds that appears to be irreversible at higher doses of cidofovir and that lower concentrations of this drug should be reserved 
for intralesional administration.

Long-term risks associated with intralesional cidofovir administration in patients with RRP have not been well studied. Overall, few adverse events have been recorded in the published reports. In a case series discussed earlier, ${ }^{84}$ two adult patients developed a cutaneous rash after a single intralesional cidofovir injection. However, whether this effect was a direct result of cidofovir administration is unclear, since the rash did not recur in either patient during subsequent cidofovir treatment. In the same series, another adult experienced headaches after each intralesional cidofovir injection that responded to symptomatic treatment. One patient complained of precordialgia after receiving three cidofovir injections. Intralesional injections in this case series were not associated with any local adverse reactions, such as inflammation, scarring, or fibrosis.

In contrast, another group of authors frequently noted a local inflammatory response at the injection site 7-14 days after each intralesional cidofovir injection. ${ }^{85}$ However, the authors stated that this response was not associated with any new areas of scarring, web formation, or impaired vibration of the vocal fold mucosa. In the case series describing treatment with percutaneous intralesional cidofovir injections, ${ }^{88}$ the only complaint patients reported was mild stinging during administration of the drug. An effect of cidofovir on complete blood cell counts and blood chemistry profiles was not

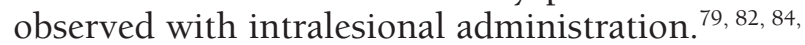
86, 88 Airway compromise during cidofovir injections was not reported in any of the case series.

Of theoretic concern is the possibility that intralesional cidofovir may lead to malignant transformation at the injection site. Although no data suggest that intravenous cidofovir is carcinogenic, subcutaneous cidofovir injections in rats were associated with development of adenocarcinoma. ${ }^{77,95}$ This effect was not observed in primates, however. ${ }^{95}$ Neoplastic changes have not been reported in any of the case series in which cidofovir was administered intralesionally or intravenously to children or adults with RRP. One case series described two patients with a diagnosis of verrucous carcinoma, but the authors ultimately felt that this disease was present before cidofovir treatment. ${ }^{84}$

The question of whether cidofovir leads to development of laryngeal carcinoma is confounded by previous observations that HPV itself may predispose patients to development of squamous cell carcinoma in both laryngotracheal and bronchoalveolar areas. Long-term follow-up of patients receiving intralesional cidofovir is necessary to fully understand the potential carcinogenicity of cidofovir therapy.

\section{Dosage and Duration of Therapy}

The optimal dose, frequency, and duration of intralesional cidofovir therapy for patients with RRP have yet to be determined. Whether a direct relationship exists between dose or duration of therapy and positive clinical outcomes is not clear. For treatment of CMV retinitis, cidofovir is administered as a $5-\mathrm{mg} / \mathrm{kg}$ infusion once/week for 2 weeks, followed by the same dose once every other week. ${ }^{76}$ Intralesional cidofovir was administered well below this dose to patients with RRP. Administration of higher doses may have contributed to improved patient outcomes in two case series. ${ }^{84,85}$ However, inclusion of adult patients in both series also may have positively influenced clinical outcomes, since the disease course is less aggressive in adults than in children.

Another study also administered higher doses of cidofovir $38-57 \mathrm{mg}$ in the adult cohort. ${ }^{88}$ However, in contrast to the two case series, ${ }^{84,85}$ none of the study patients achieved complete remission with percutaneous cidofovir treatment. Based on findings from their stepped-dose cidofovir protocol, the authors of one case series concluded that some patients may benefit from an increased dose if they do not respond to a series of cidofovir 5-mg injections. ${ }^{84}$ This gives clinicians a strategy for evolving the use of this promising treatment when initial results in a particular patient are suboptimal.

However, long-term risks associated with intralesional administration of high cidofovir doses have not been adequately studied. Administration of high cidofovir doses led to scarification of the vocal cords in a canine model $^{94}$ but has not been described in humans. Physicians and patients must balance consideration of disease severity with a degree of uncertain risk when considering escalated treatment regimens.

The frequency of injections reported in the literature also varies, with most treatments administered from every 2 weeks to once/month. For the most part, timing of cidofovir injections correlated with timing of excisional surgery. In one study, the rationale for biweekly cidofovir injections was based on the intracellular half-life 
of cidofovir's active metabolite, cidofovir diphosphate. ${ }^{79}$ The intracellular half-life of cidofovir diphosphate has ranged from 17-24 hours. Other studies have suggested that formation of another cidofovir metabolite, HPMPCp-choline adduct, with a reported intracellular half-life of 48 hours, produces a reservoir of drug from which the active metabolite is slowly released. ${ }^{69,96,97}$

In vitro, cidofovir demonstrated antiviral efficacy against HSV-1 even after discontinuation of drug treatment. ${ }^{96}$ The investigators attributed the persistence in cidofovir's antiviral efficacy to slow clearance of its metabolites from the cell. The long intracellular half-life of cidofovir allows for weekly administration during induction and every-other-week administration during maintenance therapy for CMV retinitis. One of the series described previously used a cidofovir injection frequency of every 6-8 weeks, ${ }^{82}$ compared with biweekly or monthly regimens reported by other authors. This may have contributed to the finding that only one of four patients in the series demonstrated a complete response as a result of cidofovir treatment, and it would suggest that a shorter interval between injections may be warranted.

Given the significant variability in the presentation and progression of RRP, it is unlikely a single interval between cidofovir treatments that is appropriate for all patients can be defined. Most of these injection series were performed in the operating room, using direct laryngoscopy and general anesthesia. Therefore, practical, medical, and financial factors, aside from drugrelated factors, will ultimately be determinants of the most appropriate dosage regimen and treatment approach for each patient.

Duration of therapy has also varied greatly among case series. Several patients experienced marked improvement in their severity scores and airway status. However, after discontinuation of cidofovir injections, they experienced recurrence of papillomata requiring medical intervention. In one report, only one of five patients treated with only four cidofovir injections experienced complete remission. ${ }^{79}$ Similarly, five (46\%) of the 11 patients in another study required additional treatment after the initial series of four injections. ${ }^{83}$ These patients had mild or moderate-to-advanced disease at baseline.

Basing duration and frequency of cidofovir therapy to the life cycle of HPV has also been suggested, so that treatment is administered when the virus is in its active phase, and cidofovir can interfere with viral DNA synthesis. ${ }^{83}$ However, a test that can be used in clinical practice to determine when HPV is in its active phase does not appear to be available at this time.

\section{Limitations of the Literature}

Most case series have reported positive outcomes with administration of intralesional cidofovir, and enthusiasm for its use in patients with RRP seems justifiably high. However, several questions remain as to what role cidofovir should play as an adjuvant therapy. Results of the case series are limited by small study populations as well as a lack of control groups and a blinded study design. In many of the case series and case reports, patients underwent surgical debulking or received other adjuvant therapies in addition to intralesional or intravenous cidofovir, thereby hindering the ability to attribute any positive outcomes solely to cidofovir. It remains to be seen whether the positive results in the case reports that described concomitant administration of cidofovir and interferon reflect a synergistic effect on the inhibition of HPV proliferation, as has been demonstrated in vitro. ${ }^{90,91,98}$

Furthermore, it is well established that the disease course and severity of RRP are characterized by significant interpatient and intrapatient variability. Although some patients may experience spontaneous remission, others may experience aggressive regrowth of papillomata despite repeated surgical and pharmacologic interventions. The unpredictable nature of RRP makes it difficult to evaluate the efficacy of cidofovir for treatment of the disease in a controlled setting.

Also unclear from the literature is whether cidofovir would preferentially show increased efficacy against certain HPV types, since HPV typing was not performed for all study patients. Some data suggest that infection with HPV-11 is associated with more aggressive disease than infection with HPV-6. However, viral subtypes generally have not shown substantial differences in response to different adjuvant therapies. ${ }^{82,} 99$ The literature is not clear as to whether the efficacy of intralesional cidofovir varies depending on the site of the papillomatous lesions and the ability to achieve therapeutic drug concentrations in HPV-infected cells. Successful outcomes with intralesional and intravenous cidofovir therapy have been reported in patients with localized and diffuse disease extending from laryngeal to 
tracheobronchial regions. One group of authors suggested that an inverse correlation might exist between the efficacy of cidofovir and baseline disease severity. ${ }^{83}$ However, the statistical analysis performed with their small study population demonstrated that disease severity was not predictive of a poor response to cidofovir.

\section{Conclusion}

Recurrent respiratory papillomatosis is a rare, potentially fatal disease characterized by numerous recurrences and refractoriness to surgical and various adjuvant treatments. Cidofovir, an antiviral agent with activity against HPV, the causative agent of RRP, has shown promising results for adjuvant treatment of RRP in both adults and children. In patients with mild-to-severe disease, cidofovir administered either intralesionally or intravenously has resulted in partial to complete regression of papillomata, improvement in voice quality, and decreased need for surgery. Further studies are necessary to determine the most appropriate dose, frequency, and duration of therapy, and to more fully characterize the safety profile of cidofovir administered as an intralesional injection.

\section{References}

1. Gomez MA, Drut R, Lojo MM, Drut RM. Detection of human papillomavirus in juvenile laryngeal papillomatosis using polymerase chain reaction. Medicina 1995;55:213-17.

2. Kashima HK, Kessis T, Mounts P, Shah K. Polymerase chain reaction identification of human papillomavirus DNA in $\mathrm{CO}_{2}$ laser plume from recurrent respiratory papillomatosis. Otolaryngol Head Neck Surg 1991;104:191-5.

3. Bonnez W, Reichman RC. Papillomaviruses. In: Mandell GL, Bennett JE, Dolin K, eds. Principles and practice of infectious disease. Philadelphia, PA: Churchill Livingstone, 2000:1630-44.

4. Phelps WC, Alexander KA. Antiviral therapy for human papillomaviruses: rationale and prospects. Ann Intern Med 1995; 123;368-82.

5. Hill DS, Akhtar S, Corroll A, Croft CB. Quality of life issues in recurrent respiratory papillomatosis. Clin Otolaryngol 2000;25:153-60

6. Bishai D, Kashima H, Shah K. The cost of juvenile-onset recurrent respiratory papillomatosis. Arch Otolaryngol Head Neck Surg 2000;126:935-9.

7. Reeves WC, Ruparelia SS, Swanson KI, Derkay CS, Marcus A, Unger ER. National registry for juvenile-onset recurrent respiratory papillomatosis. Arch Otolaryngol Head Neck Surg 2003;129:976-82.

8. Doyle DJ, Gianoli GJ, Espinola T, Miller RH. Recurrent respiratory papillomatosis: juvenile versus adult forms. Laryngoscope 1994;104(5 pt 1):523-7.

9. Morgan AH, Zitsch RP. Recurrent respiratory papillomatosis in children: a retrospective study of management and complications. Ear Nose Throat J 1986;65:19-28.

10. Derkay CS. Task force on recurrent respiratory papillomas: a preliminary report. Arch Otolaryngol Head Neck Surg 1995;121:1386-91.
11. Derkay CS. Recurrent respiratory papillomatosis. Laryngoscope 2001;111:57-69.

12. Puranen M, Yliskoski M, Saarikoski S, Syrjanen K, Syrjanen S. Vertical transmission of human papillomavirus from infected mothers to their newborn babies and persistence of the virus in childhood. Am J Obstet Gynecol 1996;174:694-9.

13. Smith EM, Johnson SR, Cripe TP, Pignatari S, Turek L. Perinatal vertical transmission of human papillomavirus and subsequent development of respiratory tract papillomatosis. Ann Otol Rhinol Laryngol 1991;100:479-83.

14. Tseng CJ, Liang CC, Soong YK, Pao CC. Perinatal transmission of human papillomavirus in infants: relationship between infection rate and mode of delivery. Obstet Gynecol 1998;91:92-6.

15. Shah KV, Stern WF, Shah FK, Bishai D, Kashima HK. Risk factors for juvenile onset recurrent respiratory papillomatosis. Pediatr Infect Dis J 1998;17:372-6.

16. Kashima HK, Shah F, Lyles A, et al. A comparison of risk factors in juvenile-onset and adult-onset recurrent respiratory papillomatosis. Laryngoscope 1992;102:9-13.

17. Shah K, Kashima H, Polk BF, Shah F, Abbey H, Abramson A. Rarity of cesarean delivery in cases of juvenile-onset respiratory papillomatosis. Obstet Gynecol 1986;68:795-9.

18. Sedlacek T, Lindheim S, Eder C, et al. Mechanism of human papillomavirus transmission at birth. Am J Obstet Gynecol 1989;161:55-9.

19. Tseng CJ, Lin CY, Wang RL, et al. Possible transplacental transmission of human papillomavirus. Am J Obstet Gynecol 1992;166(1 pt 1):35-40.

20. Clarke J, Terry RM, Lacey CJ. A study to estimate the prevalence of upper respiratory tract papillomatosis in patients with genital warts. Int J STD AIDS 1991;2:144-5.

21. Kramer SS, Wehunt WD, Stocker JT, Kashima H. Pulmonary manifestations of juvenile laryngotracheal papillomatosis. Am J Roentgenol 1985;144:687-94

22. Blackledge F, Anand VK. Tracheobronchial extension of recurrent respiratory papillomatosis. Ann Otol Rhinol Laryngol 2000;109:812-18.

23. Steinberg BM, Topp WC, Schneider PS, Abramson AL. Laryngeal papillomavirus infection during clinical remission. N Engl J Med 1983;308:1261-4.

24. Ferenczy A, Mitao M, Nagai N, Silverstein SJ, Crum CP. Latent papillomavirus and recurring genital warts. N Engl J Med 1985;313:784-8.

25. Kimberlin DW. Pharmacotherapy of recurrent respiratory papillomatosis. Expert Opin Pharmacother 2002;3:1091-9.

26. Cook JR, Hill DA, Humphrey PA, Pfeifer JP, El-Mofty SK. Squamous cell carcinoma arising in recurrent respiratory papillomatosis with pulmonary involvement: emerging common pattern of clinical features and human papillomavirus serotype association. Mod Pathol 2000;13:914-18.

27. Rady PL, Schnadig VJ, Weiss RL, Hughes TK, Tyring SK. Malignant transformation of recurrent respiratory papillomatosis associated with integrated human papillomavirus type 11 DNA and mutation of p53. Laryngoscope 1998;108:735-40.

28. Orphanidou D, Dimakou K, Latsi P, et al. Recurrent respiratory papillomatosis with malignant transformation in a young adult. Respir Med 1996;90:53-5.

29. Doyle DJ, Henderson LA, LeJeune FE Jr, Miller RH. Changes in human papillomavirus typing of recurrent respiratory papillomatosis progressing to malignant neoplasm. Arch Otolaryngol Head Neck Surg 1994:120:1273-6.

30. Gaylis B, Hayden RE. Recurrent respiratory papillomatosis: progression to invasion and malignancy. Am J Otolaryngol $1991 ; 12: 104-12$

31. Schnadig VJ, Clark WD, Clegg TJ, Yao CS. Invasive papillomatosis and squamous carcinoma complicating juvenile laryngeal papillomatosis. Arch Otolaryngol Head Neck Surg 1986;112:966-71

32. Sakakura A, Yamamoto Y, Takasaki T, Makimoto K, Nakamura M, Takahashi H. Recurrent laryngeal papillomatosis developing into laryngeal carcinoma with human papilloma virus (HPV) 
type 18: a case report. J Laryngol Otol 1996;110:75-7.

33. Guillou L, Sahli R, Chaubert P, Monnier P, Cuttat JF, Costa J. Squamous cell carcinoma of the lung in a nonsmoking, nonirradiated patient with juvenile laryngotracheal papillomatosis: evidence of human papillomavirus-11 DNA in both carcinoma and papillomas. Am J Surg Pathol 1991;15:891-8.

34. Helmuth RA, Strate RW. Squamous carcinoma of the lung in a nonirradiated, nonsmoking patient with juvenile laryngotracheal papillomatosis. Am J Surg Pathol 1987;11:643-50.

35. Patel N, Rowe M, Tunkel D. Treatment of recurrent respiratory papillomatosis in children with the microdebrider. Ann Otol Rhinol Laryngol 2003;112:7-10.

36. Pasquale K, Wiatrak B, Woolley A, Lewis L. Microdebrider versus $\mathrm{CO}_{2}$ laser removal of recurrent respiratory papillomas: a prospective analysis. Laryngoscope 2003;113:139-43.

37. Perkins JA, Inglis AF Jr, Richardson MA. Iatrogenic airway stenosis with recurrent respiratory papillomatosis. Arch Otolaryngol Head Neck Surg 1998;124:281-7.

38. Ossoff RH, Werkhaven JA, Dere H. Soft-tissue complications of laser surgery for recurrent respiratory papillomatosis. Laryngoscope 1991;101:1162-6.

39. Crockett DM, McCabe BF, Shive CJ. Complications of laser surgery for recurrent respiratory papillomatosis. Ann Otol Rhinol Laryngol 1987;96:639-44.

40. Wetmore SJ, Key JM, Suen JY. Complications of laser surgery for laryngeal papillomatosis. Laryngoscope 1985;95:798-801.

41. Harries ML, Juman S, Bailey CM. Recurrent respiratory papillomatosis in the larynx: re-emergence of clinical disease following surgery. Int J Pediatr Otorhinolaryngol 1995:31:259-62.

42. Shapiro AM, Rimell FL, Shoemaker D, Pou A, Stool SE. Tracheotomy in children with juvenile-onset recurrent respiratory papillomatosis: the Children's Hospital of Pittsburgh experience. Ann Otol Rhinol Laryngol 1996;105:1-5.

43. Lopez Aguado D, Perez Pinero B, Betancor L, Mendez A, Campos Banales E. Acyclovir in the treatment of laryngeal papillomatosis. Int J Pediatr Otorhinolaryngol 1991;21:269-74.

44. Endres DR, Bauman NM, Burke D, Smith RJ. Acyclovir in the treatment of recurrent respiratory papillomatosis: a pilot study. Ann Otol Rhinol Laryngol 1994;103(4 pt 1):301-5.

45. Kiroglu M, Cetik F, Soylu L, et al. Acyclovir in the treatment of recurrent respiratory papillomatosis: a preliminary report. Am J Otolaryngol 1994;15:212-14.

46. Morrison GA, Kotecha B, Evans JN. Ribavirin treatment for juvenile respiratory papillomatosis. J Laryngol Otol 1993;107:423-6.

47. Mutz I, Zoubek A, Baumgartner F. Successful bleomycin treatment of bronchopulmonary papillomatosis in a child [in German]. Monatsschr Kinderheilkd 1983;131:464-6.

48. Winnen P. Intrafocal bleomycin injection in florid oral papillomatosis (papillomatosis mucosae carcinoides) [in German]. Z. Hautkr 1983;58:733-41.

49. Mehta P, Herold N. Regression of juvenile laryngobronchial papillomatosis with systemic bleomycin therapy. J Pediatr 1980;97:479-80.

50. Harcourt JP, Worley G, Leighton SE. Cimetidine treatment for recurrent respiratory papillomatosis. Int J Pediatr Otorhinolaryngol 1999;51:109-13.

51. Leventhal BG, Kashima HK, Mounts P, et al. Long-term response of recurrent respiratory papillomatosis to treatment with lymphoblastoid interferon alfa-N1: papilloma study group. N Engl J Med 1991;325:613-17.

52. Healy GB, Gelber RD, Trowbridge AL, Grundfast KM, Ruben RJ, Price KN. Treatment of recurrent respiratory papillomatosis with human leukocyte interferon: results of a multicenter randomized controlled clinical trial. N Engl J Med 1988;319:407-13

53. Leventhal BG, Kashima HK, Weck PW, et al. Randomized surgical adjuvant trial of interferon alfa-nl in recurrent papillomatosis. Arch Otolaryngol Head Neck Surg 1988;114:1163-9.
54. Schuurman AM, van den Broek P. Results of treatment with $\alpha$ interferon in adult-onset laryngeal papillomatosis. Clin Otolaryngol 1986;11:447-53

55. McCabe B, Clark KF. Interferon and laryngeal papillomatosis: the Iowa experience. Ann Otol Rhinol Laryngol 1983;92(1 pt $1): 2-7$.

56. Haglund S, Lundquist PG, Cantell K, Strander H. Interferon therapy in juvenile laryngeal papillomatosis. Arch Otolaryngol 1981;107:327-32.

57. Lippman SM, Donovan DT, Frankenthaler RA, et al. 13-cisretinoic acid plus interferon- $\alpha 2 \mathrm{a}$ in recurrent respiratory papillomatosis. J Natl Cancer Inst 1994;86:859-61.

58. Rosen CA, Woodson GE, Thompson JW, Hengesteg AP, Bradlow HL. Preliminary results of the use of indole-3-carbinol for recurrent respiratory papillomatosis. Otolaryngol Head Neck Surg 1998;118:810-15.

59. Coll DA, Rosen CA, Auborn K, Potsic WP, Bradlow HL. Treatment of recurrent respiratory papillomatosis with indole3-carbinol. Am J Otolaryngol 1997;18:283-5.

60. Avidano MA, Singleton GT. Adjuvant drug strategies in the treatment of recurrent respiratory papillomatosis. Otolaryngol Head Neck Surg 1995;112:197-202.

61. van der Beek JM, Cremers CW, van den Broek P. Successful methotrexate therapy in oral florid papillomatosis. Clin Otolaryngol 1980;5:365-71.

62. Shikowitz MJ, Abramson AL, Freeman K, Steinberg BM, Nouri M. Efficacy of DHE photodynamic therapy for respiratory papillomatosis: immediate and long-term results. Laryngoscope 1998;108:962-7.

63. Abramson AL, Shikowitz MJ, Mullooly VM, Steinberg BM, Hyman RB. Variable light-dose effect on photodynamic therapy for laryngeal papillomas. Arch Otolaryngol Head Neck Surg 1994;120:852-5.

64. Bujia J, Feyh J, Kastenbauer E. Photodynamic therapy with derivatives from hematoporphyrines for recurrent laryngeal papillomatosis of the children. Early results [in Spanish]. An Otorrinolaringol Ibero Am 1993;20:251-9.

65. Osborne C, LeBoeuf H, Jones DV Jr. Isotretinoin in respiratory papillomatosis [letter]. Ann Intern Med 2000;132:1007.

66. Eicher SA, Taylor-Cooley LD, Donovan DT. Isotretinoin therapy for recurrent respiratory papillomatosis. Arch Otolaryngol Head Neck Surg 1994;120:405-9.

67. Bell R, Hong WK, Itri LM, McDonald G, Strong MS. The use of cis-retinoic acid in recurrent respiratory papillomatosis of the larynx: a randomized pilot study. Am J Otolaryngol 1988;9:161-4.

68. De Clercq E. Antivirals for the treatment of herpes virus infections. J Antimicrob Chemother 1993;32(suppl A):121-32.

69. Ho HT, Woods KL, Bronson JJ, De Boeck H, Martin JC, Hitchcock MJ. Intracellular metabolism of the antiherpes agent (S)-1-[3-hydroxy-2-(phosphonylmethoxy) propyl] cytosine. Mol Pharmacol 1992;41:197-202.

70. De Clercq E. Cidofovir in the therapy and short-term prophylaxis of poxvirus infections. Trends Pharmacol Sci 2002;23:456-8.

71. Johnson JA, Gangemi JD. Selective inhibition of human papillomavirus-induced cell proliferation by (s)-1-[3-hydroxy2-(phophonylmethoxy)propyl]cytosine. Antimicrob Agents Chemother 1999:43:1198-205.

72. Andrei G, Snoeck R, Schols D, De Clercq E. Induction of apoptosis by cidofovir in human papillomavirus (HPV)-positive cells. Oncol Res 2001;12:397-408

73. Abdulkarim B, Sabri S, Zelenika D, et al. Antiviral agent cidofovir decreases Epstein-Barr virus (EBV) oncoproteins and enhances the radiosensitivity in EBV-related malignancies. Oncogene 2003;22:2260-71.

74. Abdulkarim B, Sabri S, Deutsch E, et al. Antiviral agent cidofovir restores p53 function and enhances the radiosensitivity in HPV-associated cancers. Oncogene 2002:21:2334-46.

75. Van Custem E, Snoeck R, Van Ranst M, et al. Successful treatment of a squamous papilloma of the hypopharynxesophagus by local injections of (s)-1-(3-hydroxy-2- 
phosphonylmethoxypropyl)cytosine. J Med Virol 1995;45: 230-5.

76. Gilead Sciences, Inc. Vistide (cidofovir) package insert. Foster City, CA; 2000

77. Pransky SM, Magit AE, Kearns DB, Kang DR, Duncan NO. Intralesional cidofovir for recurrent respiratory papillomatosis in children. Arch Otolaryngol Head Neck Surg 1999;125:1143-8.

78. Pransky SM, Brewster DF, Magit AE, Kearns DB. Clinical update on 10 children treated with intralesional cidofovir injections for severe recurrent respiratory papillomatosis. Arch Otolaryngol Head Neck Surg 2000;126:1239-43.

79. Pransky SM, Albright JT, Magit AE. Long-term follow-up of pediatric recurrent respiratory papillomatosis managed with intralesional cidofovir. Laryngoscope 2003;113:1583-7.

80. El Hakim H, Waddell AN, Crysdale WS. Observations on the early results of treatment of recurrent respiratory papillomatosis using cidofovir. J Otolaryngol 2002;31:333-5.

81. Chhetri DK, Shapiro NL. A scheduled protocol for the treatment of juvenile recurrent respiratory papillomatosis with intralesional cidofovir. Arch Otolaryngol Head Neck Surg 2003;129:1081-5.

82. Milczuk HA. Intralesional cidofovir for the treatment of severe juvenile recurrent respiratory papillomatosis: long-term results in 4 children. Otolaryngol Head Neck Surg 2003;128:788-94.

83. Akst LM, Lee W, Discolo C, Knott D, Younes A, Koltai PJ. Stepped-dose protocol of cidofovir therapy in recurrent respiratory papillomatosis in children. Arch Otolaryngol Head Neck Surg 2003;129:841-6.

84. Snoeck R, Wellens W, Desloovere C, et al. Treatment of severe laryngeal papillomatosis with intralesional injection of cidofovir [(s)-1-(3-hydroxy-2-phosphonylmethoxypropyl) cytosine]. J Med Virol 1998;54:219-25.

85. Bielamowicz S, Villagomez V, Stager SV, Wilson WR. Intralesional cidofovir therapy for laryngeal papilloma in an adult cohort. Laryngoscope 2002;112:696-9.

86. Naiman AN, Ceruse P, Coulombeau B, Froehlich P. Intralesional cidofovir and surgical excision for laryngeal papillomatosis. Laryngoscope 2003;113:2174-81.

87. Neumann K, Pudszuhn A, Welzel C, Bartel-Friedrich S, Passmann M. Intralesional cidofovir injections for recurrent laryngeal papillomatosis: first results [in German]. Laryngorhinootologie 2003;82:700-6.

88. Chhetri DK, Blumin JH, Shapiro NL, Berke GS. Office-based treatment of laryngeal papillomatosis with percutaneous injection of cidofovir. Otolaryngol Head Neck Surg
2002;126:642-8

89. Derkay CS, Malis DJ, Zalzal G, Wiatrak BJ, Kashima HK, Coltrera MD. A staging system for assessing severity of disease and response to therapy in recurrent respiratory papillomatosis. Laryngoscope 1998;108:935-7.

90. Dancey DR, Chamberlain DW, Krajden M, Palefsky J, Albert PW, Downey GP. Successful treatment of juvenile laryngeal papillomatosis-related multicystic lung disease with cidofovir: case report and review of the literature. Chest 2000;118: 1210-14.

91. Armbruster C, Kreuzer A, Vorbach H, Huber M, Armbruster C. Successful treatment of severe respiratory papillomatosis with intravenous cidofovir and interferon alpha-2b. Eur Respir J 2001;17:830-1

92. Van Valckenborgh I, Wellens W, De Boeck K, Snoeck R, De Clercq E, Feenstra L. Systemic cidofovir in papillomatosis [online exclusive article]. Clin Infect Dis 2001;32:e62-4. Available from www.journals.uchicago.edu/CID/journal/issues/ v32n3/000253/000253.html. Accessed August 31, 2004

93. Cundy KC. Clinical pharmacokinetics of the antiviral nucleotide analogues cidofovir and adefovir. Clin Pharmacokinet 1999;36:127-43.

94. Chhetri DK, Jahan-Parwar B, Hart SD, Bhuta SM, Berke GS, Shapiro NL. Local and systemic effects of intralaryngeal injection of cidofovir in a canine model. Laryngoscope 2003;113:1922-6.

95. Center for Drug Evaluation and Research. Minutes from the joint antiviral drugs committee and ophthalmic drugs subcommittee, 1996 Mar 14-15. Available from http://www.fda. gov/cder/foi/adcomm/96/avdac_joint_031496_summmin_ac.pd f. Accessed August 31, 2004.

96. Afouna MI, Mehta SC, Ghanem AH, et al. Influence of the treatment protocol upon the in vivo efficacy of cidofovir (HPMPC) and of acyclovir (ACV) formulations in topical treatment of cutaneous HSV-l infection in hairless mice. J Pharm Sci 1999;88:530-4.

97. Aduma P, Connelly CM, Srinivas RV, Fridland A. Metabolic diversity and antiviral activities of acyclic nucleoside phosphonates. Mol Pharmacol 1995;47:816-22.

98. Johnson JA, Gangemi JD. $\alpha$-Interferon augments cidofovir's antiviral and antiproliferative activities. Antimicrob Agents Chemother 2003;47:2022-6.

99. Rabah R, Lancaster WD, Thomas R, Gregoire L. Human papillomavirus-11-associated recurrent respiratory papillomatosis is more aggressive than human papillomavirus6-associated disease. Pediatr Dev Pathol 2001;4:68-72. 